\title{
Innovations in health care services: The CAALYX system
}

\author{
Artur Rocha ${ }^{a, *}$, Angelo Martins ${ }^{a, b}$, José Celso Freire Junior ${ }^{a, c}$, Maged N. Kamel Boulos ${ }^{d}$, \\ Manuel Escriche Vicente ${ }^{e}$, Robert Feld ${ }^{f}$, Pepijn van de Ven ${ }^{g}$, John Nelson ${ }^{g}$, Alan Bourke ${ }^{g}$, \\ Gearóid ÓLaighin ${ }^{h}$, Claudio Sdogati ${ }^{i}$, Angela Jobes ${ }^{j}$, Leire Narvaiza ${ }^{k}$, Alejandro \\ Rodríguez-Molinero ${ }^{k}$
}

a Instituto de Engenharia Sistemas e Computadores do Porto, Porto, Portugal

$\mathrm{b}$ Instituto Superior de Engenharia do Porto, Porto, Portugal

c Faculdade de Engenharia de Guaratinguetá, Universidade Estadual Paulista, Guaratinguetá, SP, Brazil

d Faculty of Health and Social Work, University of Plymouth, Plymouth, Devon, UK

e Telefónica Investigación y Desarrollo, Valladolid, Spain

f Corscience GmbH \& Co. KG, Erlangen, Germany

g Department of Electronic \& Computer Engineering, University of Limerick, Limerick, Ireland

h School of Engineering \& Informatics, NUI Galway, University Road, Galway, Ireland

i COOSS Marche Onlus, Dipartimento R\&D, Ancona, Italy

j Synkronix Incorporation Ltd, Dunston House, Ipswich, UK

k Fundació Hospital Comarcal Sant Antoni Abat, Vilanova i la Geltrú, Spain

\section{A R T I C L E I N F O}

Article history:

Received 19 April 2010

Received in revised form

22 February 2011

Accepted 10 March 2011

Keywords:

Ambient Assisted Living

Elderly healthcare

Sensor network

Personal area network

Location-based system

Fall and mobility sensor

Caretaker site

Home System

Observation patterns

CAALYX

eHealth

eInclusion

\begin{abstract}
A B S T R A C T
Purpose: This paper describes proposed health care services innovations, provided by a system called CAALYX (Complete Ambient Assisted Living eXperiment). CAALYX aimed to provide healthcare innovation by extending the state-of-the-art in tele-healthcare, by focusing on increasing the confidence of elderly people living autonomously, by building on the knowledge base of the most common disorders and respective characteristic vital sign changes for this age group.

Methods: A review of the state-of-the-art on health care services was carried out. Then, extensive research was conducted on the particular needs of the elderly in relation to home health services that, if offered to them, could improve their day life by giving them greater confidence and autonomy. To achieve this, we addressed issues associated with the gathering of clinical data and interpretation of these data, as well as possibilities of automatically triggering appropriate clinical measures. Considering this initial work we started the identification of initiatives, ongoing works and technologies that could be used for the development of the system. After that, the implementation of CAALYX was done.

Findings: The innovation in CAALYX system considers three main areas of contribution: (i) The Roaming Monitoring System that is used to collect information on the well-being of the elderly users; (ii) The Home Monitoring System that is aimed at helping the elders independently living at home being implemented by a device (a personal computer or a set top box) that supports the connection of sensors and video cameras that may be used for monitoring and for interaction with the elder; (iii) The Central Care Service and Monitoring
\end{abstract}

* Corresponding author at: Information Systems and Computer Graphics Unit, INESC Porto, Campus da FEUP, Rua Dr. Roberto Frias, 378, 4200-465 Porto, Portugal. Tel.: +351 222094 199; fax: +351 222094050.

E-mail address: artur.rocha@inescporto.pt (A. Rocha).

1386-5056/\$ - see front matter @ 2011 Elsevier Ireland Ltd. All rights reserved.

doi:10.1016/j.ijmedinf.2011.03.003 
System that is implemented by a Caretaker System where attention and care services are provided to elders, where actors as Caretakers, Doctors and Relatives are logically linked to elders. Innovations in each of these areas are presented here.

Conclusions: The ageing European society is placing an added burden on future generations, as the 'elderly-to-working-age-people' ratio is set to steadily increase in the future. Nowadays, quality of life and fitness allows for most older persons to have an active life well into their eighties. Furthermore, many older persons prefer to live in their own house and choose their own lifestyle. The CAALYX system can have a clear impact in increasing older persons' autonomy, by ensuring that they do not need to leave their preferred environment in order to be properly monitored and taken care of.

(c) 2011 Elsevier Ireland Ltd. All rights reserved.

\section{Introduction}

CAALYX (Complete Ambient Assisted Living Experiment) was a two-year project partially funded by the European Commission (EC) under the Sixth Framework Programme in response to the strategic objective of eInclusion. The project had a total of eight participants in six European countries: Telefónica Investigación y Desarrollo, Spain (Coordinator), INESC Porto, Portugal, Corscience GmbH \& Co. KG, Germany, COOSS Marche Onlus, Italy, University of Plymouth, United Kingdom, Fundació Hospital Comarcal Sant Antoni Abat, Spain, Synkronix Ltd, United Kingdom and University of Limerick, Ireland.

Innovation involves the creation of a product, process, service, market or organizational structure in response to a perceived need. The demographic shift, that envisages an increase in the need to provide effective health care services for older people living in Europe is well known. A total dependency ratio of three inactive persons (elderly dependants of 65 and over, plus young dependants under 15) for every four of working age is predicted by 2050 [1]. In this scenario, the provision of effective health care services is of critical importance to the social and economic welfare of every country.

Increased health care service demands required from an already overworked health care system, has led to urgent need for effective, scalable health-care services. Consequently within EU, significant investment is being provided for research projects that would create effective health-care services. The healthcare innovations provided by CAALYX were in response to this need and specifically had 4 objectives:

1. To identify which vital signs and observation patterns are most important in determining probable critical states of an elder's health;

2. To develop a geo-located electronic device capable of measuring vital signs and detecting falls of the older person in the domestic environment and outside;

3. To provide secure monitoring of groups of individuals by a caretaker who will decide whether to communicate events identified by the system to the emergency service (112);

4. To implement a social tele-assistance service that can be easily operated by the users.

To achieve these objectives the CAALYX system comprises four subsystems:
1. Caretaker System: application that is responsible for linking all stakeholders: elders, from their Mobile and Home System, caretakers, doctors, relatives, the map server and emergency services;

2. Mobile System: responsible for controlling a Body Area Network (BAN) comprised of a set of well-being sensors and for inferring possible concerning states of the elder's health by determining relevant variations in selected physiological parameters;

3. Home System: offers elderly access to their medical data recorded by the BAN and home sensors (as well as their TV) and is the primary means of interaction between the elderly and the CAALYX system;

4. Wearable Light Device (WLD): is a key component of the BAN, merging in one package an ECG instrument, an SpO2 meter, a temperature sensor and a fall and mobility sensor. The WLD has a central role in health monitoring and in the emergency detection functionality of the system.

\section{Background}

When considering innovation it must be noted that this activity is not static; new health care services continue to be investigated, developed and implemented. There are always opportunities to innovate within any system environment. The difficult task is identifying innovations which have already occurred, or are in the process of being completed.

By carrying out a comprehensive review of health care services it is possible to identify current state-of-the-art in this domain and where innovation could be directed. Therefore the CAALYX project carried out a detailed review of this domain in order to highlight several interesting opportunities for innovation.

In this section we discuss the analyzed health care services and identify how the CAALYX project could respond in an innovative way to making these services more efficient and effective.

\subsection{Electronic patient records}

A Patient Record (PR) comprises of all the data and documents generated or received during the care of a patient at a health care organization. In its digital form an Electronic Patient Record (EPR) can be deemed a complete or partial patient 
record stored on an electronic storage medium. Electronic Patient Record systems allow the storage and manipulation of EPRs to support the continuing efficiency and quality of an integrated health service framework. Patient records are a 'pivotal' piece in the jigsaw of health care for every member of society. The challenges of managing electronic patient records within their health-care service is a challenge for innovators. A key issue is that patient data are often spread over disparate patient record systems. A dentist may store one set of information and a doctor may store another set of information. Key personal data may be stored many times, leading to excessive duplication and the potential for error.

\subsection{Data safety and security}

The design and development of an innovative health care service must address the interdependent issues of patient data safety and security. Storage and communication of patient data should be regarded by each system stakeholder, as of the utmost importance. It becomes even more critical and difficult to maintain when it can be accessed/exchanged by many other medical information systems.

National patient record systems may have to adopt sophisticated international security standards. A possible double edged sword can be envisaged: by adopting a international security standard would ensure a common security framework but it could also lead to a potential global breach of data security if the standard implemented is found to be a source of weakness. Strong authentication, encryption and digital signatures based on public key infrastructures are examples of security methods being used to ensure data security.

Personal information may be captured and manipulated for many different reasons. It is vital that the information is effectively protected and reliably available. By adopting a 'code of good practice', the methods and required security standards can be identified, described and followed in order to effectively manage patient data security. The code of good practice would be based on current EU legal requirements, relevant standards and professional best practice. A security code of good practice, if followed would protect collected patient data by providing safe, secure and reliable use of technology to promote patient healthcare. Personal data security is vital, if users are to maintain trust and confidence in a technical solution.

\subsection{Integration and interoperability}

The effort directed towards the implementation of international standardization for the different components that comprise a health care service, continues. Integration and interoperability are key factors that need to be considered by innovators of health care services. The basic requirement of patient data exchange needs to be addressed if the data captured by one medical information systems can be sent and utilized by another. Health information system standards for health data exchange include Health Level Seven $\left(\mathrm{HL}^{1}\right)$ version 3 and the related Clinical Document Standard

\footnotetext{
${ }^{1}$ HL7 is one of several American National Standards Institute (ANSI)-accredited Standards Developing Organizations (SDOs) operating in the healthcare arena.
}

(CDA). Semantic interoperability surpasses the technicalities of patient record exchange, using several methods. It is clear that the standardization of a health care service needs to be addressed at several different levels, including basic data types, messages, services and clinical terminologies.

An innovator of a health care service needs to identify and adopt the relevant standards required within the context of their work. Doing so increases the prospect for creating a health care service that is innovative, exploitable and future proof.

\subsection{Health sensors}

In the tele-health market there are several systems available that offer wireless remote monitoring of patients. Those medical systems usually consist of a tele-health record application on a server and a home-based system acting as a relay station and communication facility. A set of sensors is connected to this device via cable or wireless connections.

Sensors utilized are typically off-the-shelf devices that measure one specific parameter [2] like blood pressure, SpO2, ECG or heart rate. A closer look shows that most are still designed for one time measurements rather than continuous monitoring or transmission of vital signs. In most cases, devices present the recorded data "as is" without sophisticated analysis or the possibility of a prompt review by a medical expert.

In the area of emergency call systems for elderly people at home, most systems are designed around a device that has a "Help" button to be pressed by the user or a simple fall detection sensor that triggers an alarm. These systems are usually not connected to tele-medical systems and can therefore not make use of any additional medical sensors that the person is using.

\subsection{Fall and mobility monitoring}

In fall monitoring, there is a gap between technology used in research and commercially. The latter tend to err on the side of safety and therefore employ algorithms detecting the user orientation, sometimes in combination with impact detection. These systems results in high sensitivity (likelihood of detecting a fall correctly) for falls but a limited specificity (likelihood of triggering a false fall alert). Research into fall detection focuses on more elaborate algorithms, in which velocity, the occurrence of several impacts or the timing of certain events are used to improve the specificity of the fall detection algorithms.

Mobility monitoring is generally limited to around four states: walking, standing, lying and sitting. Most of the algorithms found, rely on the use of at least two sensors and some of the algorithms employ sensors in the user's environment to improve the identification of activities undertaken. In general, portable mobility monitoring devices target a limited number of activities.

\subsection{Opportunities for innovation}

Considering the general points presented in the context of the CAALYX project the following possible areas for innovation were established: 
- Scalability: As the number of patients continues to grow, the finite set of health care professionals must be able to use any developed system effectively in order to cater for a growing system demand. A health care system that allows a health professional to oversee a large (growing) number of patients could make an exploitable, cost/benefit business case [3];

- Vital sign monitoring: The identification/evaluation and proactive treatment of the older person, may allow health care to be provided quickly i.e. 'illness prevention is better than cure';

- Patient record exchange: The adoption of identified international standards would prevent CAALYX becoming another 'island' of patient data in a sea of medical information interchange;

- Patient data privacy/security: Effective security methods and processes need to be established, in order to provide a health care service security framework;

- Online doctor visits: A scalable technology-based health care system that would allow effective use of health professionals (who could even be based outside the patient's home country);

- Event driven, adaptable and scalable monitoring infrastructure: this infrastructure will allow for the health professionals to easily configure software/virtual sensors for each user through some observation patterns using the set of available real and virtual sensors, i.e., the user's BAN, sensors at home, the Mobile System (including geopositioning) and user health data at a caretaker server and, eventually, health/medical records.

In this paper we present how some of these possibilities have been implemented in the CAALYX system.

\section{Methods and materials}

This paper is based on the knowledge, expertise and experience obtained by the different research groups and practitioners who actively participated in the CAALYX project. The original idea for the project was to define an innovative system of tele-health care/monitoring that allows elderly people to live autonomously in a comfortable and safe way.

To achieve this goal, initial research was carried out to identify opportunities for innovation that could be applied to such systems. In parallel, a user scenario was devised and a thorough user requirements capture process was conducted. Options were highlighted and validated by end-users.

Considering this initial work, research about elder health data and how to reason with it was conducted. Finally, subsystems required for CAALYX were set out and the implementation of the system took place.

Briefly, the 4 stages of the methodology are: (i) research about innovation possibilities in the area of health care systems (presented in Section 2); (ii) research of elder health data (Section 3.1); (iii) analysis of initiatives and techniques that could support the development of CAALYX (Section 3.2); (iv) implementation of the system (Section 4).

\subsection{Elder health data}

The review has focused on CAALYX specific target group - the elders, analysing on-going medical research with this patient group to narrow the scope of CAALYX technical research, thus increasing the effectiveness of the system.

\subsubsection{The problem domain: reasoning with clinical} measurements

Effective monitoring of a person's health first requires one to gather all relevant information about his/her health; and then correctly interpreting such information in order to reach conclusions about the person's health state. This process is not straight-forward. A number of factors must be taken into account in order to reach valid conclusions about a person's health, all of which require the gathering of vast amounts of information. Furthermore, no fixed unequivocal rules exist for interpreting such information; several correct interpretations are possible, which lead to different conclusions. Deciding which interpretation best conveys the actual health state of a person often requires additional information or observing the course of events (observing health data changes over time is one of the most reliable methods of reaching a valid conclusion).

The interpretation process requires an analysis of the available data in order to decide whether they correspond to a normal health state or to evidence of a health alteration. Accurate assessment of a person's health status requires the interpretation of different types of information, which we classify into three categories:

- Retrospective information: refers to the known health background of the involved person (in the clinical practice, it corresponds to the personal background information in the medical record).

- Transversal information: refers to the person's health state at the moment of examination. It consists of several elements: symptoms, signs, etc.

- Prospective information: refers to health changes subsequent to examination. This may support or reject the conclusions reached during examination.

Considering the clinical measurement and from the clinical point of view, we decided that the CAALYX system needs to have two essential functions:

- Autonomous detection of health alterations: it is capable of detecting health alterations and triggering alarms without human intervention (the system interprets the available information). The system can gather transversal information from several sensors, as well as prospective information by carrying out intensive follow up measurements if necessary. Additionally, the system "interprets" this information autonomously, before triggering an alarm. Thus, alarms are only activated when necessary, and accompanied by helpful information for the diagnosis.

- Gathering of information: the system provides physicians and caretakers with detailed information about the person's health state. To that end, CAALYX gathers rich and diverse transversal information, both from sensors and 
questionnaires, and offers it together with prospective and retrospective information, so that the users can figure out the health state of the monitored elderly person.

\subsubsection{Information gathering: sensors and questionnaires for monitoring the elderly}

Monitoring an elderly person's health requires a large amount of information, but the monitoring system can only feature a limited number of measuring devices.

Since the profile of the diseases affecting elderly persons is different from that for younger persons, as is the impact of diseases on their health and quality of life, we designed a structured process aimed at selecting the most sui sensors to be used with the elderly population, which constitutes a remarkable innovation. After that, devices providing the greatest amount of information have been selected.

For the CAALYX project, we decided to monitor the persons' health status through a number of measuring devices, several of which are multifunctional body sensors capable of detecting different changes and measuring certain physiological parameter values. In addition, questionnaires were also used to gather subjective information and data that cannot be measured by sensors.

\subsubsection{Automated interpretation: simulating clinical reasoning}

The automated interpretation of elder's health data through simulation of clinical reasoning constitutes an interesting research problem.

The CAALYX approach to this problem was based on a powerful tool capable of providing enough information about an elderly person's health status for a health-provider to properly assess out the situation from a remote location. Moreover, the system can autonomously interpret part of the information, so as to trigger an alarm if the user's health gets worse. The solution to this problem is one of the most important CAALYX innovations.

CAALYX also uses rules for interpreting a person's health state. These rules are based on three major aspects: normal limits of the physiological parameters, simultaneous observation of several physiological parameters and temporal evolution of physiological parameters. Thus, detecting a physiological parameter value beyond the normal limits will not trigger alarm provided that other parameters are not altered or that the detected alteration is transient.

The limits within which physiological parameter values should be considered normal in the elderly population are not well known, although some data are available about the limits of blood pressure [4-6] and heart rate [7-9]. We were unable to find well-controlled studies about the limits of peripheral blood oxygen saturation, body temperature and respiratory rate in the elderly. However, clinical tradition and knowledge acquired by geriatricians worldwide through medical assistance support the idea that the normal value range for these parameters in the elderly is different from that of younger persons.

Therefore, as part of the medical research component of the CAALYX project, a national-level study was conducted on a representative sample of the Spanish elderly population, which for the first time established the limits that should be considered normal in this population, thus providing a guide for the system to interpret data.

The Spanish study was conducted in a sample of 800 older-than-sixty-four years. The sample was gathered using a probabilistic cluster multistage sampling, and it was stratified according sex, age and size of the habitat (rural town communities, cities and big cities), additionally it represented all the geographical areas of Spain. All the stratum were defined to reproduce the proportions estimated in the population, except the age stratum which was intentionally non-proportional, aiming to over-represent the characteristics of the very old population (over than 80 years old), which is the one with the most marked differential features with respect to the younger population.

This study found marked differences in the normal ranges of respiratory rate and body temperature compared to younger populations (preliminary data published $[10,11]$ ).

Hence, the interpretation of elderly persons' health data by the CAALYX system were based on normal limits that have been established by this project for the first time. This also constitutes a remarkable innovation, which makes the system specifically aimed at monitoring elderly persons' health and thus is different from any other available system.

The data interpretation by the system is guided by observation patterns (see Section 3.2.2 for details), which references two types of normal limits: absolute and relative. Absolute limits are only surpassed by major changes of a physiological parameter, which immediately trigger an alarm without possible inhibition by other incoming data. Relative limits may be surpassed by moderate changes, which can be considered normal or abnormal, according to their duration in time, the values of other physiological parameters, or the intensity of the user's motor activity at the time of measurement.

The system's response in the face of mild changes was established by the physicians on the CAALYX system on the basis of clinical reasoning, so that not all alterations are equally important or trigger the same response. The clinical rules were based on experts' opinion, who defined the actions to be performed by the system considering all the possible findings that the system was capable of detecting taking into account the possible combinations of data from sensors. The observation and alarm responses are pre-established according to the possible diagnosis behind such alterations and are intended to adjust to the most appropriate action in every case.

The observation patterns that guide the system in case of mild changes increase the system's sensitivity to detect health alterations (not to overlook changes with latent manifestations) and prevents the triggering of false alarm when isolated out-of-limits values are detected. This automated health-data interpretation system, based on normal limits that have been established for elderly people and rules that emulate clinical reasoning, makes the CAALYX system unique and different from any other monitoring system.

\subsection{State of the art}

This section summarizes the state of the art on each of the four major CAALYX subsystems, namely: the Caretaker System; 
the Mobile System; the Home System; and the Wearable Light Device.

\subsubsection{Caretaker System}

The idea of a Caretaker System has been proposed in several projects. Basic tele-assistance/tele-care services on the market support emergency situations when users are at home. They are based on regular land-lines. The emergency device normally has a push button to trigger emergency calls/alerts. A state of the art was carried out on current European projects. The most similar to the CAALYX propositions are:

- MobiHealth [12]: funded under 6th Framework program, offered a mobile healthcare service permitting health professionals to remotely assess, diagnose and treat patients while they are free to continue with daily life activities and stay fully mobile. It added sensors interconnected under a Body Area Network managed by a PDA or mobile telephone;

- Dreaming [13]: funded by the European Commission under the new Competitiveness and Innovation Program (CIPPSP), the main aim is keeping elderly people in their home environment as long as their physical and mental conditions allow this. The Dreaming project intends to create technological solutions and social assistant services to pursue this objective;

- Persona [14]: funded under 6th Framework Program, aimed at developing a system architecture that would provide the interoperability of services and applications and could be easily adopted to the specific requirements of each user according to the following scenarios: Multimedia Experience Sharing, Daily Life Manager, Surveillance and Risk Management, and Personal Mobility Management.

These projects present several overlapping features with CAALYX like, development of new mobile valued-added services in the area of health care and providing the possibility of patients to be fully mobile while undergoing health monitoring (MobiHealth); monitoring achieved with a Home System in elderly home and video conference services (Dreaming).

The European Commission has also funded other projects to foster Ambient Assisted Living for example INHOME [15], EMERGE [16] and SOPRANO [17] offering of telemedicine and social functionalities, such as Teleconference with doctors and friends and outdoor User tracking functionality using GPS in the mobile monitoring systems (PERSONA).

The most important difference between CAALYX and the solutions presented are:

- MobiHealth: unlike CAALYX, this system is not specifically designed for elderly people but focuses on a greater variety of scenarios to confirm technical viability of GPRS and UMTS communications services to support health services; GPS and fall detection are not available;

- Dreaming: the set of sensors is different both in the mobile monitoring system and the Home System (the mobile monitoring system does not have any medical sensors attached, and the Home System has ambient and health sensors); the health sensors are activated by the elderly person;

- Persona: there is no automatic alarm detection or decision support functionalities based on the medical information, the Personal Health Management features do not work in a real-time paradigm but it has a lot of use cases that regard assistive technologies for the daily living of the elderly person.

\subsubsection{Mobile System}

The concept of Wireless Body Area Network has been used in several initiatives and projects related with health monitoring $[18,19]$ as previously discussed (Section 3.2.1). Additionally, location in health-care services has also been previously addressed [20].

However, the CAALYX Mobile System has been designed with the aim of reducing the dependency from a central system, thus contributing to minimizing power consumption by communicating only when strictly necessary, which also helps in protecting the elder's privacy in terms of sensitive data such as health status and location.

Furthermore, interoperability has been a primary goal when developing the mobile application framework. CAALYX leverages on the effort of the Open Geospatial Consortium (OGC) community [21], particularly on the Sensor Web Enablement (SWE) workgroup [22] as a way of establishing a foundation for a "plug-and-play" sensor network.

It is worth mentioning that although a set of recommendations and good practices has been extracted from the outcomes of this workgroup [23], the full set of standards was not adopted since it would not be efficient when applied to the sensor protocols used in CAALYX. This seems, however, the correct research approach because on one hand there is a harmonization effort between the OGC SWE standardization initiative and the smart transducers family of standards (IEEE 1451) which seems more appropriate for CAALYX sensors and on the other hand, even if sensors in a Body Area Network are not geographically distributed, measurements and observations performed by resorting to this sensor network tend to be, which will facilitate the introduction of geographic context awareness.

CAALYX is built upon the same notion of Observation as defined by Fowler [24]. Since an Observation is quantification about a given phenomenon, it makes sense to transport the concept to the medical domain as observations make it possible to associate specific conditions (e.g. traits) and measurements with people at a given point in time. We have discovered that this had already been suggested by Yoder et al. [25] when designing software for the Illinois Department of Public Health.

The same author also proposed an adaptive [26] modelling style in order to cope with the vast quantity of possible traits and measurements in this domain. Although the authors also introduce the concept of composite observation in [25], the need of defining a Template for such Observations or incorporating the knowledge from a decision-tree as done in CAALYX is never mentioned.

\subsubsection{Home System}

For the Home System subsystem, the state of the art was also established by a comparison with similar projects. Numerous projects related with Ambient Assisted Living were analyzed, such as AMIGO (Ambient intelligence for the networked home environment) [27], EPERSPACE (Towards the era of personal 
services at home and everywhere) [28] and CHS (Distance Information Technologies For Home Care) [29] just to name a few.

Among these projects, AMIGO is probably the closest to CAALYX given the fact that an open platform is considered. AMIGO was developed from September 2004 to February 2008 and is an integrated project which researched and developed an open, standardized, interoperable platform for a networked Home System, as well as user services, improving end-user usability and attractiveness.

The developed middleware was designed to ensure automatic dynamic configuration of the devices and services. Thus, third parties can use the platform, to deploy services and content for the networked home more easily and quickly, resulting in a faster market introduction. The project would create and demonstrate prototype applications that improve everyday life, addressing all the vital user aspects such as: home care and safety, home information and entertainment, and extension of the home environment by means of personal communication.

\subsubsection{Wearable Light Device}

The process of taking measurements usually requires a lot of user interaction because typically sensors are not designed to be worn over the whole day and in order to record more than one vital sign several sensors have to be used one after the other.

One example of this is the system offered by Zydacron which consists of a Home-system called Carestation which can be connected to a set of sensors. Measurements are performed on the initiative of the user.

An example for a system implementing tele-medical heart monitoring is the Paxiva service offered by Philips Telemedicine Service which uses an electrocardiogram (ECG) device called Viapac to record and transmit a 12 channel ECG. For each recording the device is put on by the user and after the measurement transmission of the recorded data over the phone line is manually triggered.

An example of a system intended for use in a clinical environment is the Drager Infinity M300. Similarly to the WLD it consists of an ECG and Pulseoximeter in one device and can transmit the vital signs wirelessly. Consistent with its intended use in a clinical environment it is designed to be operated by medical personnel rather than by the patient. Patients wear it round the neck in a clear waterproof bag. The vital signs are transmitted wirelessly to a proprietary server system.

The HCG-801-E ECG device from Omron is an example of a device that does not perform a sophisticated analysis of collected data. It can be used to record a single channel ECG at home. After the recording is complete a simple analysis is performed. The data can be exported from the device by taking out the SD Memory card and read its contents with a PC.

The professional range of products includes several devices designed to be integrated in a tele-medical system and sensors for measuring blood pressure, peak-flow, weight, SpO2 and ECG are typically available in those systems. Solutions for round the clock monitoring of patients are typically for use in a clinical environment and therefore are designed as a bedside-monitor. They are too bulky and heavy to carry over a long period of time and are therefore generally not used at home.

3.2.4.1. Fall and mobility sensor. The fall and mobility sensor is a subsystem of the WLD, but due to its complexity and importance it deserves to be treated separately.

Falls in the elderly are a major problem for today's society. Approximately one third of all community-dwelling elderly and $50 \%$ of nursing home residents over the age of 65 , experiences at least one fall every year [30,31]. Two thirds of elderly fallers experience a further fall within six months [31]. Older adults are hospitalized for fall-related injuries five times more often than they are for injuries from any other cause [32].

In 1987 the Kellogg international working group on the prevention of falls in the elderly defined a fall as "unintentionally coming to ground, or some lower level not as a consequence of sustaining a violent blow, loss of consciousness, sudden onset of paralysis as in stroke or an epileptic seizure" [33].

Noury [34-36], from the University of Grenoble-France, designed an autonomous sensor module, attached under the armpit, which comprised accelerometers, inclinometers and a vibration sensor. The sensor module detected when the velocity of the movement exceeded a specific threshold, the sequence from a vertical posture to the lying posture, as well as the absence of movements after the fall. The device was first tested on 5 persons who performed 15 fall scenarios 5 times and was shown to achieve a sensitivity and specificity close to $85 \%$. This is a very good fall-detection system, and performed very well in testing. The evaluation and decision algorithms are quite effective and the idea of going from acceleration, to inclination, to rate of change of inclination; to activity classification is a very effective method.

Recently, Bourke [37-39] proposed fall algorithms separately based on thresholds on both signals from a tri-axial accelerometer [37] a biaxial gyroscope [39] and a combination of both tri-axial accelerometer and gyroscopes sensors [38] and reported a performance of $100 \%$ sensitivity and specificity from both.

Kangas et al. [40] evaluated different low-complexity fall detection algorithms, using tri-axial accelerometers attached at the waist, wrist, and head. Fall data was recorded from three middle-aged subjects who performed nine intentional fall types in three directions (forward, backward, and lateral). High sensitivities (97-98\%) and specificities (100\%) were achieved in this study with quiet low-complexity algorithms, however only a small subject group $(n \leq 3)$ performing very low numbers of scripted ADL and no long-term unsupervised testing was performed.

Kangas et al. [41] also continued the evaluation of the algorithm set on data recorded by a group of 20 middle-aged (40-65 years old) volunteers performing (six different falls each performed twice) and four scripted Activities of Daily Living (ADL), also these same ADL were also recorded from 21 older people (aged 58-98 years) from a residential care unit. He showed that a simple algorithm of impact detection followed by posture measurement can discriminate various types of falls from ADL with a sensitivity of $97.5 \%$ and a specificity of $100 \%$. However only a modest amount of ADL was recorded and no long-term unsupervised recording was performed. 
Chao et al. [42] recorded tri-axial accelerometer data from the chest and waist of seven young healthy male subjects as they performed falls as well as functional normal and dynamic activities including jumping. They examined the use of acceleration cross-product and magnitude as well as post fall posture and developed and tested algorithms of varying complexity. The various algorithms were $d$ under the following categories; threshold selection, sensor placement and postfall posture (PP). Results showed that a fall detection algorithm based on the data from the chest shows better global accuracy. With post-fall posture detection leading to lower false alarm ratios, a number of commercial fall detection devices are also currently available, including:

- Tunstall ${ }^{2}$ : detect falls when the subject is not in an upright position and an impact occurs;

- Health watch, ${ }^{3}$ InTouch Medical alarm systems ${ }^{4}$ : both devices use the same algorithm, waking from standby mode if a rapid change in a person's physical orientation is detected. The process continues if an impact is then detected. Devices continue monitoring for $6-15 \mathrm{~s}$ to verify if a fall has occurred. If the person's body position remains unchanged for up to $15 \mathrm{~s}$, then a fall-alert is raised;

- $\mathrm{VRI}^{5}$ : designed to detect falls, abnormal body movements, or extended periods of inactivity and automatically summon assistance without end-user intervention, it employs accelerometers to perform this.

All of the above fall detection systems are based or use the fall detection algorithm developed by Doughty et al. [43] and Williams et al.[44]. They suggest using a two-stage detection process which senses shock and the orientation of the wearer to determine if a fall has occurred. However the thresholds for the orientation angles or time windows or impact have not been detailed in any research.

\subsection{CAALYX approach}

This section summarizes the approach employed on each of the four major CAALYX subsystems.

\subsubsection{Caretaker System}

The Caretaker System within the CAALYX system resides in the server side, where services are provided. The Caretaker System is the subsystem where attention and care services are provided to elders; where system users such as Caretakers, Doctors and Relatives are logically linked to elders. This connection is possible by taking advantage of current communication networks. Supporting services used by the Caretaker System are map services, to obtain maps, and emergency services, to request help, in case of emergency.

Health monitoring and alerts are the main concepts around which the Caretaker System has grown. Measurement of vital signs is the key process to detect medical conditions and,

\footnotetext{
2 http://www.tunstall.co.uk.

3 http://www.health-watch.com.

4 http://www.pioneermed.com.

5 http://www.monitoringcare.com.
}

therefore, to raise alerts. The elder's position is only disclosed to the caretaker in case of alert, so that a proper request to emergency services can be launched. Likewise, a communication tool to contact and verify the elder's health self-perception is needed in case of alerts.

Yearly trials have been a decisive factor in focusing effort on building a Caretaker System which is useful and meaningful for real users. Their feedback on each iteration prototype was taken advantage of to enrich the design of the next-iteration prototype. Considering these factors, the Caretaker System strategy was to provide the necessary functionalities for the trials by using leading edge technologies, architectures and standards, while meeting project deadlines.

\subsubsection{Mobile System}

The Mobile System is an important integration point in the CAALYX system, taking on the role of an "informed intermediary" between the sensors in the elder's BAN and the other CAALYX subsystems such as the Caretaker System. Not only does it provide interfaces to interact with these components, it also incorporates enough domain knowledge to act as a first level of decision making concerning the relevance of changes detected in sensor data.

For the purpose of implementing the CAALYX prototypes, a special purpose language, inspired on the SensorML OGC standard, was created to implement the domain knowledge. The language has been encoded in XML format and the structure enclosed in this XML file controls the execution of the mobile application, determining from which sensor(s) to read, the threshold levels for the selected physical signs and a decisiontree which may encompass operations (e.g. comparisons) and that may trigger other sensor readings. This XML structure has been designated "Observation Template" because the result of its execution is a "Health Observation".

Several "Observation Templates" may exist, depending on the BAN configuration and on the desired monitoring patterns/conditions. In this sense, the software framework developed for the Mobile System is said to be adaptive. Typically, a caretaker would select a pre-defined "Observation Template" from the Caretaker System and customize it for a particular elder according to his/her requirements.

An instance of an "Observation Template" has been designated "Observation Pattern" and this is the main vehicle for the Caretaker System to control the monitoring conditions for a specific elder. In the future, the caretaker may fine tune the parameters enclosed in an "Observation Pattern" with the results of a learning mechanism occurring on the server, or even remotely request the execution of a new "Observation Pattern" in order to further examine the health status of an elder.

When an "Observation Pattern" is executed by the Mobile System, whether triggered by an off-limits sensor reading or by a scheduled process, the result is a "Health Observation", which has enough semantics to be classified according to its seriousness level and may result in an "Alarm". Alarms are sent to the Caretaker System along with the "Health Observation" that triggered it. Enclosed in a "Health Observation" are the respective sensor data and location information.

It should be stressed that the CAALYX system is not limited to the use of hardware sensors, as an observation pattern 
may use the results of several other observation patterns. This mechanism was used to allow the system to be context aware, i.e. to use previous known information about the user status (location, activity level, etc.) to further improve the accuracy of the health monitoring mechanism. A very simple example would be the monitoring of an elder while jogging and at rest. The system identifies the activity level and the user location (GPS) and uses different health parameters for each situation.

From the technological point of view, the Mobile System uses an Event-Driven Architecture (EDA) [45] in order to help minimize power consumption [46] and as a natural extension to a Service Oriented Architecture (SOA). In other words, the software reacts only when relevant state changes occur (for example, the arrival of an event triggered by a sensor), leading to further actions, otherwise it will do nothing. Although this type of architecture can be used with "continuous reading" devices (such as a regular ECG device), it produces the best results in conjunction with smart devices such as the WLD. EDA is also deemed very appropriate to interface with a SOA like the one used in the Caretaker System because an event often results in the invocation of a Web Service.

\subsubsection{Home System}

The CAALYX system is complemented with a Home Monitoring/Control System aimed at helping the elders to independently live at home. This device (a personal computer or a set top box) supports the connection of Bluetooth enabled sensors (weighting scale and blood pressure meter) and video cameras that may be used for monitoring and for interaction with the elder. This device use the existing TV set to interact with the user, thus reducing the technical complexity of the device as well as reducing its cost. Using the TV and (at least) a camera, the elder may establish live connections with carers, family and friends, thus reducing isolation syndrome and other psychiatric disorders.

CAALYX has taken a user-centric approach where the user constraints and usability of the Home System have taken priority over delivery of a large set of functionalities.

\subsubsection{Wearable Light Device}

The CAALYX system is designed to meet the following requirements: continuous health monitoring, autonomous medical event detection and reaction and simple operation [47].

Those requirements suggest certain design choices for the WLD. In order to be able to meet all demands that exist for a system supporting and monitoring elderly people the features of current systems were included and extended.

Continuous health monitoring requires the sensors of the system to be active round the clock. In order to cope with the enormous amount of data that is recorded from this monitoring, a mechanism for data reduction is required.

The transmitted data must be reduced to focus the attention of the medical professional on the relevant parts and to save energy as transmission is a very expensive operation in terms of energy consumption.

To match these requirements the WLD was designed to include a number of sensors and functionalities. It integrates the following sensors into a compact casing:
- ECG, 1 channel (internally for analysis 24 Bits $500 \mathrm{~Hz}$, externally at reduced rate and resolution to save transmission bandwidth).

- Pulseoximeter for measuring Blood Oxygen Saturation (SpO2) and Photoplethysmograph (PPG).

- Temperature sensor for measuring the body surface temperature.

- Fall and Mobility Sensor (see Section 3.3.4.1 for details).

The aforementioned sensors deliver the following primary vital signs: ECG, SpO2, PPG and Temperature. Heart rate is extracted in a redundant way from the ECG and PPG. From the ECG and PPG signals, by applying the concept of sensor fusion, the Pulse Transit Time (i.e. the time the pulse wave travels from the heart to the SpO2 sensor at the user's finger) is derived. The joint analysis of the PTT, ECG and PPG allows for the extraction of the Respiratory Rate (RR) in a robust way.

As the information in these signals contains redundancy, it is possible to calculate the RR even if only the ECG is available. By attaching the Pulseoximeter, the reliability can be further improved. The fall sensor transparently transmits the fall and mobility data through the shared Bluetooth interface.

In order to simplify the handling and improve usability, the WLD was designed for ease of operation having just one button (on/off), batteries that can be recharged in the device by use of a docking station, the status (battery level, errors) are automatically reported via Bluetooth, ECG electrodes and Pulseoximeter finger-clip are easily connected and disconnected with one plug.

The technical implementation includes a highly integrated PCB design and a Bluetooth compliant interface.

The concept for the WLD includes a powerful real-time analyser of the recorded data. The analysis detects relevant patterns present in the signals and immediately reports them to the Mobile System. Additionally, certain measurements can be requested from the device.

The novel respiration rate detection algorithm, integrated in the design during the CAALYX project, is able to measure the respiration rate by signal analysis from the ECG and PPG signals.

To reduce the amount of data transmitted between the WLD and Mobile System the WLD has a number of configurable thresholds for the monitored vital signs. When a threshold is exceeded the WLD immediately reports a medical event.

Some of those events, that require an immediate reaction, trigger the transmission of an alarm signal to the caretaker without any user interaction. The caretaker can request further measurements to assess the situation and decide to alert a family member or call an ambulance. The immediate availability of detailed sensor information can improve the quality of the decisions made and therefore reduce false alarms and ensure correct recognition of critical situations.

The availability of independent vital signs enables a context aware interpretation of the results by the system. This awareness reduces false alarms and increases reliability.

3.3.4.1. Fall and mobility sensor. The fall and mobility sensor, which is integrated into the WLD, located at the subject's waist, attached either using a waist belt or a modified 
mobile-phone carry-case. It is based on measuring the accelerations of the user close to their centre of gravity, in six degrees of freedom. Using thresholds, both in acceleration, posture and in time, an algorithm determines whether a fall has occurred. Using the referred thresholds it examines a number of different kinematic, temporal and mechanical quantities, which include measuring the downward acceleration of the faller, the impact experienced by a faller, the time difference between the downward acceleration and the body impact as well as examining the orientation of the subject following the impact to see if they are lying or not.

By examining the magnitude of the downward acceleration and the impact acceleration measured at the subjects' waist, the time difference between them as well as examining the subjects' orientation following this episode, a fall can be determined by comparing these measurements to experimentally derived thresholds. These thresholds were obtained from recording 10 young health subjects performing falls and normal ADL and 10 elderly health subjects performing normal ADL in their own homes. This algorithm was inspired through examining the profile of the 3D accelerometer wave from previous indigenous research [37] as well as implementing the view that a person downward speed is also a key determinant of a fall $[48,49,38,50]$. Also, as a result of the CAALYX phase 1 trials in Ancona Italy a number of false alarms occurred [51]. These occurred when subjects performed a number of normal ADL such as: subjects sitting down in a car, lying down on a bed and falling into a sitting position on the bus. The alterations to the fall algorithm have thus been implemented to reduce the false positives due to these types of movements.

If the acceleration, impact and temporal thresholds are exceeded then a fall towards the ground followed by an impact is deemed to have occurred. The subjects' posture is then monitored to see if they are lying or standing for the following $2 \mathrm{~s}$. If the subject is lying following a fall plus a large impact then a fall-alert is registered. Upon detection of a fall-alert, the sensor will send a fall alert message to the mobile phone using Bluetooth. These messages will be repeated at specified intervals until a message is received from the phone indicating that the fall alert message has been received. Meanwhile, the fall sensor will monitor the user for a further $5 \mathrm{~min}$. In case the user has not recovered from the fall, a long-lie event will be send to the phone, thus indicating that help is required. Again, the "long-lie event" message will be repeated until the phone acknowledges having received the message. In case the user recovers from the fall, a fall recovery message is sent using the same message protocol. The algorithm developed for the CAALYX project incorporates many of the best and most reliable features of modern fall detection algorithms but it is also capable of operating on a real-time embedded system which the user wears.

\section{Technical innovation in CAALYX}

This section summarizes the technical advances on each of the five major CAALYX subsystems. Each sub-section highlights the main current and future innovation aspects.

\subsection{Caretaker System}

The technical innovations related to the Caretaker System encompass several areas and can be summarized by:

- Artificial Intelligence module to help decision-making on alerts: CAALYX' system is designed as a reactive system. Continuous health monitoring is carried out with mobile sensors while wearing the WLD. When thresholds are triggered, reactive behaviour is switched on by activating observation patterns, which intensify monitoring. These observation patterns may end by sending an alert to the Caretaker System when a health condition is observed, which will result in further actions undertaken by the different actors involved: physicians, relatives or emergency services. When the caretaker has an incoming alert, there is need to take two decisions: firstly, whether or not the alert is accepted, and secondly, which actor will be responsible for this alert. To assist in making these decisions the CAALYX Caretaker System features an Artificial Intelligence Module, which is a Bayesian Network capable of learning from previously service alerts and capable of giving probabilities to the incoming alerts. It is understood probabilities are a useful tool to help take decisions;

- User interface for physicians: CAALYX system, besides being reactive, is also proactive. Unlike the caretaker who represents reactive behaviour, the physician has a proactive attitude in the care of the elder's health. This user interface has been designed and implemented to allow the physician to have easy control on alert thresholds, health measurements and questionnaires, treatments and health record. Alerts are available for awareness and video-conference is used to hold diagnostic conversations so that the physician can complete their diagnosis of the elder's health;

- Context awareness: the CAALYX system respects elder privacy. Therefore, the elder's location is unknown by the caretaker. However, this rule is broken in two cases: when an alert happens and when the elder is at home. Geographical position is sent within the alert so that the elder can be rescued. The system informs the caretaker if he is available at the mobile phone or at the Home System, this way it provides an efficient way to contact the elder.

\subsection{Mobile System}

From the functional point of view, the most important innovation brought by the CAALYX's Mobile System component, is the reasoning capability on the mobile phone that provides simultaneous local reasoning and decision making and a sensor process orchestration system, which allows the custom definition of processes to collect information.

Such features, improve the CAALYX system in two different levels: Customization and Behaviour.

From the customization point of view, such features allow the integration of medical knowledge in the decision processes and the seamless adaptivity of the system for each user in several ways, such as thresholds, schedules and sensors.

From the behaviour point of view, the fact that the Mobile System is able to reason about the medical information and 
distinguish between the relevant health states, improves the whole CAALYX system scalability and effectiveness, since the health professional can manage more elders, and it also saves resources such as the data transfer ratio and battery life time.

Also, the fact that the information regarding a medical observation is aggregated is very important from the point of view of the usability of the CAALYX system, as doctors can better evaluate the health status for each elderly person.

From the technology perspective, CAALYX Mobile System builds upon an adaptive and extensible software framework which uses standards such as Bluetooth and Web Services to interface with the sensors and the Caretaker System, respectively. In doing so, CAALYX has moved towards an adaptive $[25,26]$, "plug-and-play" style architecture in which there is a reduced effort to integrate new sensors.

Concepts such as measurement, observation [52] and process [23] adopted in the Sensor Web Enablement (SWE) initiative by the OpenGeospatial Consortium (OCG) have inspired the management and extraction of data from a sensor network such as the BAN. Such concepts have been adapted in order to be able to work with physical signs.

The concept of observation template has been introduced and helps in improving automation in the Mobile environment. This concept is adaptable and context aware, since it supports dynamic process definition and includes a portable process engine able to interpret logical premises in order to make situation reasoning.

A further enhancement could be the implementation of a cross-environment middleware using the same principles since it would allow an automated distributed decision chain, in the sense that observation patterns could be initiated by a home sensor and then transferred to the mobile environment and vice-versa.

A recurring problem in services relying on GPS is the failure to establish a GPS fix in difficult environments [53]. In CAALYX, an Assisted GPS (A-GPS) firmware update for the Nokia N95 [54] helps the device to get a fix on GPS satellites faster and more reliably, ensuring smoother GPS functionality.

However, a more definitive workaround would be to adopt more robust geo-positioning solutions combining multiple geo-positioning technologies (cell phone, WiFi and IP address data triangulation) to cover those locations where conventional GPS alone would fail. Some existing services and technologies such as Google My Location ${ }^{6}$ or Geosense ${ }^{7}$ for Windows 7 offer this capability[55]. Such a hybrid, "intelligent" solution should be able to automatically and transparently select and switch between multiple geo-positioning methods as necessary to ensure uninterrupted location functionality, compensating for the inherent deficiencies of a GPS-only solution, as well as working in tandem with it in those smart phones with a built-in GPS sensor.

\subsection{Home System}

One of the main obstacles in the take up of Tele-care type systems has been the fear of technology by the elderly and their

\footnotetext{
${ }^{6}$ http://www.google.com/mobile/maps.

7 http://geosenseforwindows.com.
}

concerns of relying on and operating technology on which their life might depend.

During our investigation we discovered the popularity of Nintendo's Wii controller and its success among many age groups especially children and older generation where a very simple controller with very few buttons on the controller is used to control user interaction on the TV screens. The fact that the controller is Bluetooth enabled offers a major plus point for the CAALYX project where it is used as a means of communication with the Home System device.

Working closely with a group of elderly in UK, an intuitive graphical interface was developed and tested for use on TV. The Controller was complemented by this interface where choices are highlighted in an explicit manner to avoid any confusion in respect to what the user needs to achieve. This approach is linked to the simple navigational method (which is used in many remote controls) to allow users to navigate options through arrow keys.

Furthermore in the CAALYX project it was decided to use the MythTV platform in order to integrate TV functionality with the display of medical data. To this end a new interaction mechanism was developed to allow ease of use of the system and switching between the TV and the medical system at the touch of a button.

This resulted in the development of an easy to use interface allowing easy navigation of the system using very few buttons.

\subsection{Wearable Light Device}

The WLD includes several new concepts that enable the CAALYX system to fulfil its requirements in monitoring elderly people.

By the integration of several sensors in one device the usability was greatly increased compared to systems that consist of a number of single sensors as there is only one device to recharge, don and operate.

By having all sensors integrated in one device there is only one radio connection and one energy source which leads to a better energy efficiency. Usability and reliability are also greatly enhanced by only having one device to recharge and handle. Only these features enable the CAALYX system to perform constant vital sign monitoring in elderly people at a high level of usability.

A second important innovation is the reduction of the data that needs to be transmitted by using advanced ECG analysis algorithms. The reduction of data results in an increased energy efficiency of the system which makes it possible to realize smaller and lighter devices and longer operation.

The WLD is the first wearable device to include a state of the art respiration rate detection algorithm, measured by analysis of the ECG and PPG signals. In contrast to mechanical methods for measuring the respiration rate this approach does not include extra cables, sensors or belts around the patient's chest which are very inconvenient for constant monitoring.

The real-time analysis of ECG in the CAALYX system introduces the possibility to not only passively detect cardiac events but to react to those events without any user interaction. This means an essential advance in security for the users of the device. 
The ease of handling and operation of the device enables use by patients without any support from medical personal.

The technical innovations related to fall and mobility sensors include the following:

A flexible accelerometer based fall and mobility sensor has been designed which can easily interface with any Bluetooth enabled device. The hardware architecture is based on a low power microprocessor which allows for autonomous operation for extended periods of time. The software architecture allows for the use of several different fall and mobility algorithms and this functionality has been used to test and validate various fall and mobility detection algorithms. The new algorithms developed for the CAALYX project show high specificity and sensitivity in tests performed with real subjects $[56,57]$.

Due to the development and use of an extensive message protocol, it is trivial to replace the current Bluetooth wireless connection with any other sort of connection. For example in the main CAALYX trials, messages were delivered to the WLD through a UART, after which the WLD forwarded these messages to the higher layers of the CAALYX system. The protocol supports core functions such as the reporting of fall alerts, long-lie events, fall recoveries, ADL reports, and raw data reports. In addition, the protocol messages support configuration, calibration, and debugging of the device.

Through the integration of the fall and mobility sensor with the rest of the CAALYX platform, the system is equipped with the unique capability to validate and plan measurements; as previously mentioned. For example in the case that a high heart rate is measured, an alarm should not be raised if it is known that the elderly user is performing, or has just performed, vigorous activity. Conversely, the system can take into account periods of inactivity to schedule measurements that would be influenced, by physical the activity of the user.

\section{Conclusion}

This paper presents the main achievements and innovations from the CAALYX project. The general principle underlying the project was to extend the elder's autonomy, making it easier for the elder to interact with the environment, thus improving his/her daily life. This main objective and the complementary ones were fulfilled, in summary:

- Build and deploy a prototype of the Light Wearable Device with fall and mobility sensor and a set of body vital signs (ECG, heart rate, respiratory rate, temperature and pulse oximetry);

- Build and deploy a prototype of the mobile phone/data logger to manage the elder's Body Area Network (BAN) that manages communications with the caretaker site efficiently;

- Build and deploy a prototype of the caretaker site, including tools for elder management and received data visualization;

- Build and deploy a prototype of the home environment with its architecture, sensing capabilities and elder data display and navigation;

- Test the above system components in a real environment subject to the assessment of actual end-users, thus proving
Summary points

What is already developed in the area:

- A broad range of sensors, usually working in an isolated fashion, destined to medical monitoring during a short-term period;

- Alert services, typically initiated by the use of a panic button, establishing direct communication with an emergency service;

- A few body-worn sensor prototypes communicating with a server mostly on a continuous basis;

- Studies about typical values and limit conditions for some vital signs, for the general population.

What this project added to our knowledge:

- Typical values and thresholds for the selected vital signs, specific for the elderly population. This includes relative limits, which could be meaningless when considered isolated, but in conjunction with other measurements and background/context information may help to infer potentially dangerous situations for the elder health;

- A Roaming Monitoring System autonomously run by Observation Patterns, which encode the aforementioned domain knowledge (fine-tuned to an individual), using it to act as an informed local intermediary in order to trigger alarms and initiate communication with a caretaker only when necessary;

- A configurable Body Area Network, composed by a set of smart sensors (packaged together for usability reasons) capable of generating alerts according to pre-defined conditions and of being reprogrammed on-the-fly by a sensor controller (e.g. mobile phone);

- A fall and mobility sensor, implementing an improved fall detection algorithm and auto-calibration on startup;

- A complete, scalable and flexible system for autonomous elders, capable of using data provenient from roaming monitoring devices and home sensors, complementing it with information gathered by questionnaires in order to help caretakers to devise potentially dangerous deviations to the elder's health status.

its capability to impact and break the balance of the current welfare system for elderly persons.

\section{Authors' contributions}

JCFJ conceived and drafted the manuscript as he joined the INESC team at the end of the project. Other authors contributed unique insights and technical know-how in their respective areas of expertise, and revised and approved the final manuscript. 


\section{Conflict of interest statement}

None declared.

\section{Acknowledgement}

The CAALYX project (Complete Ambient Assisted Living Experiment) is funded by the Commission of the European Union as part of its Sixth Framework Programme (IST-2005045215).

\section{REFERE N C E S}

[1] R. McDougall, Too many people: Europe's population problem. URL

http://www.optimumpopulation.org/opt.europe.html, 2009 (cited 24 January 2010).

[2] H.J. Lee, S.H. Lee, K.-S. Ha, H.C. Jang, W.-Y. Chung, J.Y. Kim, Y.-S. Chang, D.H. Yoo, Ubiquitous healthcare service using zigbee and mobile phone for elderly patients, International Journal of Medical Informatics 78 (3) (2009) 193-198, URL http://www.sciencedirect.com/science/article/B6T7S4TB0V91-1/2/74c4d80285003417ea92316705997dd5.

[3] D. Konstantas, An overview of wearable and implantable medical sensors, in: IMIA Yearbook of Medical Informatics 2007, no. 46, Schattauer Publishers, 2007, pp. 66-69.

[4] K. Björklund, L. Lind, H. Lithell, Twenty-four hour ambulatory blood pressure in a population of elderly men, Journal of Internal Medicine 248 (6) (2000) 501-510.

[5] C. Suárez, C. del Arco, T. Sáez, F. Blanco, J.M. Ruiz, M. Alonso, R. Gabriel, Ambulatory blood pressure monitoring in the aged. The EPICARDIAN Study. The EPICARDIAN Work Group, Revista española de cardiología 51 (12) (1998) 956-971.

[6] J.R. Banegas, F. Rodriguez-Artalejo, L.M. Ruilope, A. Graciani, M. Luque, J.J. Cruz-Troca, R. Garcia-Robles, J. Tamargo, J. Rey-Calero, Hypertension magnitude and management in the elderly population of Spain, Journals of Hypertension 20 (2002) 2157-2164, Lippincott Williams \& Wilkins.

[7] H. Tasaki, T. Serita, A. Irita, O. Hano, I. Iliev, C. Ueyama, K. Kitano, S. Seto, M. Hayano, K. Yano, A 15-year longitudinal follow-up study of heart rate and heart rate variability in healthy elderly persons, Journals of Gerontology Series A: Biological Sciences and Medical Sciences 55 (2000) 744-749, The Gerontological Society of America.

[8] J.M. Ribera, A.J. Cruz, J.L. Zamorano, F. Pérez-Casar, Cardiac rate and hyperkinetic rhythm disorders in healthy elderly subjects: evaluation by ambulatory electrocardiographic monitoring, Gerontology 35 (2-3) (1989) 158-164.

[9] A. García, M. Valdés, V. Sánchez, F. Soria, A. Hernández, T. Vicente, F. Pérez, P. Rodriguez, Cardiac rhythm in healthy elderly subjects, The Clinical Investigator 70 (2) (1992) 130-135.

[10] J.M.S. Rodríguez, J.M.N. Fontiveros, L.N. Grau, A.Y. Marco, A. Rodríguez-Molinero, Distribution of basal respiratory frequency and peripheral-blood oxygen saturation in spanish elders without cardio-respiratory pathology, 50 Congreso de la Sociedad Española de Geriatría y Gerontología, Granada, España, June, 2008.

[11] M.D.D. Díaz, F.C. Ricker, L.N. Grau, A.Y. Marco, A. Rodríguez-Molinero, Normal distribution of oral temperature values in spanish elders, $50^{\mathrm{a}}$ Congreso de la Sociedad Española de Geriatría y Gerontología, Granada, España, June, 2008.
[12] E.E. AB, Innovative GPRS/UMTS Mobile Services for Applications in Healthcare. URL http://130.89.10.26/mhealth/, 2010 (cited 25 January 2010).

[13] Dreaming elDeRly-friEndly alarm handling and monitoring. URL http://www.dreaming-project.org/, 2008 (cited 26 January 2010).

[14] PERSONA PERSONA: perceptive spaces promoting independent aging. URL http://www.aal-persona.org, 2010 (cited 25 January 2010).

[15] I. Project NHOME Project - an intelligent interactive services environment for assisted living at home. URL http://www.ist-inhome.eu, 2008 (cited 26 January 2010).

[16] E. Commission Emergency Monitoring and Prevention. URL http://www.emerge-project.eu, 2010 (cited 26 January 2010).

[17] E. Commission SOPRANO - service-oriented programmable smart environments for older Europeans. URL http://www.dreaming-project.org/, 2010 (cited 26 January 2010).

[18] E. Jovanov, A. Milenkovic, C. Otto, P. Groen, A wireless body area network of intelligent motion sensors for computer assisted physical rehabilitation, Journal of NeuroEngineering and Rehabilitation (2005) 2:6, BioMed Central Ltd.

[19] C.I.C. ETB Ltd Healthy Aims. URL http://www.healthyaims.org, 2008 (cited 26 January 2010).

[20] I. Maglogiannis, S. Hadjiefthymiades, Emerloc: location-based services for emergency medical incidents, International Journal of Medical Informatics 76 (10) (2007) 747-759, URL http://www.sciencedirect.com/science/article/B6T7S4KSVGCH-1/2/e47de592ed8a631bd0599442c80cb0a1.

[21] I. Open Geospatial Consortium, Making location count. URL http://www.opengeospatial.org, 2010 (cited 26 January 2010).

[22] I. Open Geospatial Consortium, Making location count sensor web enablement WG. URL

http://www.opengeospatial.org/projects/groups/sensorweb, 2010 (cited 26 January 2010).

[23] M. Botts, G. Percival, C. Reed, J. Davidson, OGC sensor web enablement: overview and high level architecture. URL http://www.opengeospatial.org/pt/06-046r2, 2006 (cited 26 January 2010).

[24] M. Fowler, Patterns of Enterprise Application Architecture, Addison-Wesley Professional, 2002.

[25] J.W. Yoder, F. Balaguer, R. Johnson, From analysis to design of the observation pattern. URL

http://www.joeyoder.com/Research/metadata/Observation/ ObservationModel.pdf, 2002 (cited 26 January 2010).

[26] J.W. Yoder, R. Johnson, The adaptive object model architectural style, in: Proceeding of the Working IEEE/IFIP Conference on Software Architecture 2002, 2002.

[27] AMIGO Amigo - ambient intelligence for the networked home environment. URL

http://www.hitech-projects.com/euprojects/amigo/, 2008 (cited 25 February 2010).

[28] E. GmbH, ePerSpace - towards the era of personal services at home and everywhere. URL http://www.ist-eperspace.org/, 2004 (cited 25 February 2010).

[29] N. Maglaveras, V. Koutkias, I. Chouvarda, D.G. Goulis, A. Avramides, D. Adamidis, G. Louridas, E.A. Balas, Home care delivery through the mobile telecommunications platform: the citizen health system (chs) perspective, International Journal of Medical Informatics 68 (1-3) (2002) 99-111, URL http://www.sciencedirect.com/science/article/B6T7S47BXBTX-B/2/adae7afb38df76d454688f0a654a9cc7.

[30] N. Noury, A smart sensor for the remote follow up of activity and fall detection of the elderly, in: International Conference of the IEEE Engineering in Medicine and Biology Society, Madison, WI, 2002.

[31] M.C. Nevitt, S.R. Cummings, S. Kidd, D. Black, Risk factors for recurrent non-syncopal falls. a prospective study, Journal of 
American Medical Association 261 (1989) 2663-2668.

[32] B.H. Alexander, F.P. Rivara, M. Wolf, The cost and frequency of hospitalisation for fall-related injuries in older adults, American Journal of Public Health 82 (7) (1992) 1020-1023.

[33] Kellogg International Working Group, The prevention of falls in later life, Danish Medical Bulletin 34 (Suppl. 4) (1987) $1-24$

[34] N. Noury, P. Barralon, G. Virone, P. Boissy, M. Hamel, P. Rumeau, A smart sensor based on rules and its evaluation in daily routines, in Proceedings of the 25th Annual International Conference of the IEEE, Engineering in Medicine and Biology Society, vol. 4, pp. 3286-3289, 2003.

[35] N. Noury, A smart sensor for the remote follow up of activity and fall detection of the elderly, 2nd Annual International Conference on Microtechnologies in Medicine and Biology (2002) 314-317, doi:10.1109/MMB.2002.1002337.

[36] N. Noury, T. Herve, V. Rialle, G. Virone, E. Mercier, G. Morey, A. Moro, T. Porcheron, Monitoring behavior in home using a smart fall sensor and position sensors, in IEEE-EMBS Microtechnologies in Medicine \& Biology (2000) 607-610, doi:10.1109/MMB.2000.893857.

[37] A. Bourke, J. O’Brien, G. Lyons, Evaluation of a threshold-based tri-axial accelerometer fall detection algorithm, Gait Posture 26 (2) (2007) 194-199.

[38] A.K. Bourke, K.J. O'Donovan, G.M. OLaighin, The identification of vertical velocity profiles using an inertial sensor to investigate pre-impact detection of falls, Medical Engineering \& Physics 30 (9) (2008) 937-946.

[39] A.K. Bourke, G.M. Lyons, A threshold-based fall-detection algorithm using a bi-axial gyroscope sensor, Medical Engineering \& Physics 30 (2008) 84-90.

[40] M. Kangas, et al., Comparison of low-complexity fall detection algorithms for body attached accelerometers, Gait Posture 28 (2) (2008) 285-291.

[41] M. Kangas, et al., Sensitivity and specificity of fall detection in people aged 40 years and over, Gait Posture 29 (4) (2009) 571-574.

[42] P.K. Chao, et al., A comparison of automatic fall detection by the cross-product and magnitude of tri-axial acceleration, Physiological Measurement 30 (10) (2009) 1027-1037.

[43] K. Doughty, R. Lewis, A. McIntosh, The design of a practical and reliable fall detector for community and institutional telecare, J Telemed Telecare 6 (Suppl. 1) (2000) 150-154.

[44] G. Williams, K. Doughtyt, K. Cameront, D.A. Bradley, A smart fall \& activity monitor for telecare applications, in: Proceedings of the 20th Annual International Conference of the IEEE Engineering in Medicine and Biology Society, 1988, pp. 1151-1154

[45] B.M. Michelson, Event-driven architecture overview, Patricia Seybold Group. URL http://www.omg.org/soa/Uploaded\% 20Docs/EDA/bda2-2-06cc.pdf, February 2006 (cited 27 February 2010).
[46] H. Muller, C. Randell, Workshop on Software Engineering for Wearable and Pervasive Computing, Limerick, Ireland, June, 2000

[47] R. Steele, A. Lo, C. Secombe, Y.K. Wong, Elderly persons' perception and acceptance of using wireless sensor networks to assist healthcare, International Journal of Medical Informatics 78 (12) (2009) 788-801, Mining of Clinical and Biomedical Text and Data Special Issue. URL http://www.sciencedirect.com/science/article/B6T7S4X49N36-1/2/3a01e4db9f134a64724530fe07bbb811.

[48] G. Wu, S. Xue, Portable preimpact fall detector with inertial sensors, IEEE Transactions on Neural and Rehabilitation Systems Engineering 16 (2) (2008) 178-183.

[49] G. Wu, Distinguishing fall activities from normal activities by velocity characteristics, Journal of Biomechanics 33 (11) (2000) 1497-1500.

[50] A.K. Bourke, K.J. O’Donovan, J. Nelson, G.M. OLaighin, The identification of vertical velocity profiles using an inertial sensor to investigate pre-impact detection of falls, in: Annual International Conference of the IEEE Engineering in Medicine and Biology Society, Vancouver, Canada, 2008, pp. 2832-2835.

[51] A.K. Bourke, P.W. van de Ven, A.E. Chaya, G.M. OLaighin, J. Nelson, The identification of vertical velocity profiles using an inertial sensor to investigate pre-impact detection of falls, in: Conference Proceedings - IEEE Engineering in Medicine and Biology Society, Vancouver, Canada, 2008, pp. 2844-2847.

[52] S. Cox, Observations and measurements. Part 1. Observation schema, Tech. rep., OGC, December 2007.

[53] M.N. Boulos, A. Rocha, A. Martins, M. Vicente, A. Bolz, R. Feld, I. Tchoudovski, M. Braecklein, J. Nelson, G. Laighin, C. Sdogati, F. Cesaroni, M. Antomarini, A. Jobes, M. Kinirons, Caalyx: a new generation of location-based services in healthcare, International Journal of Health Geographics 6 (9), doi:10.1186/1476-072X-6-9. URL http://www.ij-healthgeographics.com/content/6/1/9.

[54] Nokia, About assisted gps. URL http://www.nokia.co.uk/support/product-support/nokian95/a-gps, 2007 (cited 9 March 2010).

[55] Microsoft Developer Network Introduction to the windows sensor and location platform. URL

http://msdn.microsoft.com/enus/library/dd317677(VS.85).aspx, 2010 (cited 9 March 2010).

[56] A. Bourke, P. van de Ven, A. Chaya, G. OLaighin, J. Nelson, The design and development of a long-term fall detection system incorporated into a custom vest for the elderly, in: Conference Proceedings IEEE Engineering in Medicine and Biology Society, 2008, pp. 2836-2839.

[57] Bourke, P. van de Ven, A. Chaya, G. OLaighin, J. Nelson, Testing of a long-term fall detection system incorporated into a custom vest for the elderly, Conference Proceedings IEEE Engineering in Medicine and Biology Society, 2008. 